\title{
CONFORMAL TRANSFORMATIONS IN RIEMANNIAN AND HERMITIAN SPACES
}

\author{
BY KENTARO YANO
}

Communicated by S. Bochner, April 20, 1960

The purpose of the present note is to show that the results recently announced by S. I. Goldberg [1] in this Bulletin are valid also in slightly more general forms.

1. Consider a conformal Killing vector $v^{h}$ in an $n$-dimensional Riemannian space. Then the Lie derivative of the fundamental tensor $g_{j i}$ and that of Christoffel symbols with respect to $v^{h}$ are respectively given by

$$
\mathfrak{R}_{v} g_{j i}=\nabla_{j} v_{i}+\nabla_{i} v_{j}=2 \phi g_{j i}
$$

and

$$
\mathfrak{R}_{v}\left\{\begin{array}{c}
h \\
j i
\end{array}\right\}=\nabla_{i} \nabla_{i} v^{h}+K_{k j i}^{h} v^{k}=A_{j}^{h} \phi_{i}+A_{i}^{h} \phi_{j}-\phi^{h} g_{j i},
$$

where $\nabla_{j}$ is the symbol of covariant differentiation, $K_{k j l}{ }^{h}$ the curvature tensor, $A_{j}^{h}$ the unit tensor and $\phi_{i}=\nabla_{i} \phi, \phi^{h}$ being its contravariant components.

For a skew-symmetric tensor $w_{i_{p} i_{p-1} \ldots i_{1}}$, we have in general [5]

$$
\mathfrak{R}_{v} \nabla_{j} w_{i_{p}} \cdots i_{1}-\nabla_{j} \mathfrak{R}_{v} w_{i_{p}} \cdots i_{1}
$$

$$
=-\left(\mathfrak{R}_{v}\left\{\begin{array}{c}
t \\
j i_{p}
\end{array}\right\}\right) w_{t i_{p-1} \cdots i_{1}}-\cdots-\left(\mathfrak{R}_{v}\left\{\begin{array}{c}
t \\
j i_{1}
\end{array}\right\}\right) w_{i_{p} \cdots i_{2} t}
$$

Taking the skew-symmetric part with respect to $j, i_{p} \cdots i_{1}$, we find

$$
\left.\left.\mathfrak{R}_{v} \nabla_{[j} w w_{i_{p}} \cdots i_{1}\right]=\nabla_{[j} \mathfrak{R}_{v} w_{i_{p}} \cdots i_{1}\right]
$$

from which

THEOREM 1.1. The Lie derivative of a closed skew-symmetric tensor is closed.

Transvecting (1.3) with $g^{j i_{p}}$ and taking account of (1.1) and (1.2), we get

$$
\begin{aligned}
\mathfrak{l}_{v} g^{j i} \nabla_{j} w_{i i_{p-1}} \cdots i_{1} & +2 \phi g^{i i} \nabla_{j} w_{i i_{p-1}} \cdots i_{1}-g^{i i} \nabla_{j} \mathfrak{R}_{v} w_{i i_{p-1}} \cdots i_{1} \\
& =(n-2 p) \phi^{t} w_{t i_{p-1}} \cdots i_{1}
\end{aligned}
$$


from which

THEOREM 1.2. The Lie derivative of a coclosed skew-symmetric tensor of order $p$ with respect to a conformal Killing vector is coclosed if and only if $p=n / 2, n$ being even, or $\nabla^{t}\left(\phi w_{t i_{p-1}} \cdots i_{1}\right)=0$, that is, $\phi w_{i_{p}} \cdots i_{1}$ is also coclosed, where $\phi$ is the function appearing in $\&_{v} g_{j i}=2 \phi g_{j i}$.

Combining Theorems 1.1 and 1.2 we have

TheOREM 1.3. The Lie derivative of a harmonic tensor w of order $p$ in an n-dimensional Riemannian space with respect to a conformal Killing vector is also harmonic if and only if $p=n / 2, n$ being even, or $\phi w$ is coclosed.

The most specific statement resulting is as follows, see $[4 ; 5 ; 6]$.

THEOREM 1.4. The Lie derivative of a harmonic tensor w of order $p$ in an n-dimensional compact orientable Riemannian space with respect to a conformal Killing vector is zero if and only if $p=n / 2, n$ being even, or $\phi w$ is coclosed where $\phi$ is a function appearing in $\Re_{v} g_{j i}=2 \phi g_{j i}[1]$.

2. In an almost complex space, a contravariant almost analytic vector is defined as a vector $v^{h}$ which satisfies

$$
\mathfrak{Q}_{v} F_{i}{ }^{h}=v^{t} \partial_{t} F_{i}{ }^{h}-F_{i}{ }^{t} \partial_{t}{ }^{h}{ }^{h}+F_{t}{ }^{h} \partial_{i} v^{t}=0 .
$$

In an almost Hermitian space, (2.1) may be written as

$$
\ell_{v} F_{i}{ }^{h}=v^{t} \nabla_{t} F_{i}{ }^{h}-F_{i}{ }^{t} \nabla_{t}{ }^{h}+F_{t}{ }^{h} \nabla_{i} v^{t}=0,
$$

from which, by a straightforward calculation,

$$
\nabla^{i} \nabla_{i} v^{h}+K_{i}{ }^{h} v^{i}-F_{i}{ }^{h}\left(\Omega_{v} F^{i}\right)-\frac{1}{2} F_{j i}{ }^{h}\left(\Omega_{v} F^{j i}\right)=0,
$$

where $K_{i}{ }^{h}$ is the Ricci tensor and

$$
\begin{gathered}
F^{i}=\nabla^{j} F_{j}{ }^{i}, \\
F_{j i h}=\nabla_{j} F_{i h}+\nabla_{i} F_{h j}+\nabla_{h} F_{j i} .
\end{gathered}
$$

If we put

$$
S^{j i}=g^{j t}\left(\Re_{v} F_{t}^{i}\right),
$$

and suppose that the space is compact, we have 


$$
\begin{aligned}
\int\left[\left\{\nabla^{i} \nabla_{i} v^{h}+K_{i}{ }^{h} v^{i}-F_{i}{ }^{h}\left(\Omega_{v} F^{i}\right)-\frac{1}{2} F_{j_{\imath}}{ }^{h}\left(\Omega_{v} F^{j i}\right)\right\} v_{h}\right. & \\
& \left.+\frac{1}{2} S^{j i} S_{j i}\right] d \sigma=0,
\end{aligned}
$$

$d \sigma$ being volume element of the space.

From (2.3) and (2.4) we have

THEOREM 2.1. A necessary and sufficient condition for a vector $v^{h}$ in a compact almost Hermitian space to be contravariant analytic is (2.3).

Suppose that a conformal Killing vector $v^{h}$ satisfies

Substituting

$$
F_{i}{ }^{h}\left(\Omega_{v} F^{i}\right)+\frac{1}{2} F_{j i}{ }^{h}\left(\Omega_{v} F^{j i}\right)=0 .
$$

$$
\nabla^{i} \nabla_{i} v^{h}+K_{i}^{h} v^{i}=-\frac{n-2}{n} \nabla^{h}\left(\nabla_{i} v^{i}\right)
$$

obtained from (1.2) into (2.4), we find

$$
\int\left[\frac{n-2}{n}\left(\nabla_{i v^{i}}\right)^{2}+\frac{1}{2} S^{i i} S_{j i}\right] d \sigma=0,
$$

from which, for $n>2$,

$$
\nabla_{i} v^{i}=0, \quad S_{j i}=0
$$

and consequently $v^{h}$ is a Killing vector $[4 ; 6]$ and at the same time a contravariant almost analytic vector, and for $n=2$, we have $S_{j i}=0$. Thus we have

THEOREM 2.2. If a conformal Killing vector $v^{h}$ in an n-dimensional compact almost Hermitian space satisfies

$$
F_{i}{ }^{h}\left(\Omega_{v} F^{i}\right)+\frac{1}{2} F_{j i}{ }^{h}\left(\Omega_{v} F^{j i}\right)=0,
$$

then, for $n>2$, it defines an automorphism of the space, that is, the infinitesimal transformation $v^{h}$ does not change both the metric and the almost complex structure of the space, and for $n=2$, it is contravariant almost analytic.

An almost Hermitian space in which $F_{i}=0$ is satisfied is called an almost semi-Kählerian space. In such a space, we have 


$$
F_{j i h} F^{j i}=2 F_{t} F_{h}{ }^{t}=0 .
$$

Thus from Theorem 2.2, we have

THEOREM 2.3. If a conformal Killing vector $v^{h}$ in an $n(>2)$ dimensional compact almost semi-Kählerian space satisfies

$$
F_{j i h}\left(\Omega_{v} F^{j i}\right)=0 \text { or }\left(\Omega_{v} F_{j i h}\right) F^{i i}=0,
$$

then $v^{h}$ defines an automorphism in the space.

An almost Hermitian space in which $F_{j i h}=0$ is satisfied is called an almost Kählerian space. In such a space, we have

$$
F_{h}=-\frac{1}{2} F_{j i t} F^{j i} F_{h}{ }^{t}=0
$$

that is, $F_{j i}$ is harmonic. Thus from Theorem 2.3, we have

TheOREM 2.4. A conformal Killing vector $v^{h}$ in an $n(>2)$ dimensional compact almost Kählerian space defines an automorphism of the space $(c f .[1 ; 2 ; 3])$.

\section{BIBLIOGRAPHY}

1. S. I. Goldberg, Conformal transformations of Kähler manifolds, Bull. Amer. Math. Soc. vol. 66 (1960) pp. 54-58.

2. A. Lichnerowicz, Géométrie des groupes de transformations, Paris, 1958.

3. S. Tachibana, On almost analytic vectors in almost Kählerian manifolds, Tôhoku Math. J. vol. 11 (1959) pp. 247-265.

4. K. Yano, On harmonic and Killing vector fields, Ann. of Math. vol. 55 (1952) pp. $38-45$.

5. - Theory of Lie derivatives and its applications, Amsterdam, 1957.

6. K. Yano and S. Bochner, Curvature and Betti numbers, Annals of Mathematics Studies, vol. 32, 1953.

UNIVERSITY OF HONG KONG 